SLAC-TN-14-001

Replaces: SLAC CN-72

\title{
Kicker Magnet and Pulser
}

\author{
By: Fatin Bulos \\ SLAC, National Accelerator Laboratory, Stanford, California 94309 USA
}

\begin{abstract}
:
The SLC Project utilizes several fast kicker magnets. Their requirements vary somewhat, however, the cooling rings kickers have the most stringent requirements. In this note we describe the design of the positron ring kickers, and the reasons that led to it.
\end{abstract}




\section{TITLE: kicker Magnet and Pulser}

The SLC Project utilizes several fast kicker magnets. Their requirements vary somewhat, however, the cooling rings kickers have the most stringent requirements. In this note we describe the design of the positron ring kickers, and the reasons that led to it.

\section{$\underline{\text { Requirements }}$}

The positron cooling ring will have two circulating bunches $~ 50 \mathrm{n} . \mathrm{S}$. apart in time. The kicker must kick one of the bunches accurately without disturbing the other. The cooling ring itself is compact, and space is at a premium. Timing and space impose the following requirements on the kicker and its pulser.

1) The kick angle $\leq 8 \mathrm{mr}$ at $1.2 \mathrm{GeV}$. The rep. rate is $180 \mathrm{c} / \mathrm{s}$.

2) The field must rise and fall back to zero in $<100$ n.s. The peak field should be stable to better than $.1 \%$. For this reason a flat top of at least 5 n.s. width is highly desirable.

3) The total length of the magnet must be $<40 \mathrm{cms}$. Nothing at all can protrude beyond this.

\section{Magnet Design}

The above requirements led me to believe that a ferrite loaded transmission line magnet would be the best choice. The reasons are:

1) It could have a definite impedance which does not depend on the magnetic length. Hence except for requirements on filling time, length and electric field are complimentary.

2) A "square pulse" from a pulser of the same impedance would be ideal for driving the magnet.

3) Cables of the same impedance would also be ideal for connecting the pulser to the magnet. Within reason these cables could be fairly long. Such cables can also connect the magnet to its termination.

After some experimentation with ferrite loaded transmission lines, a set of rules for the design of such a magnet were established. 
Appendix Al describes in detail the design procedure. Table AI gives the results for two magnet geometrics. The appendix also gives a brief discussion of the effect of transit time.

The method of Appendix A was used to design and build a prototype magnet. An existing wire chamber cable pulser was adapted to drive the magnet at 2-6 $\mathrm{kV}$. A further modification allowed us to drive the magnet at $10 \mathrm{kV}$. This set up was used for 4 purposes.

(1) Measuring the magnet impedance (by matching) in order to compare with the calculated impedance. Checked fairly well.

(2) Studying the various methods of voltage doubling. (Two possible schemes are usable).

(3) Measuring the magnetic field and comparing with calculation. (Checked fairly we11).

(4) Studying various problems with pick up and learning how to measure the stability of the pulser and field.

In addition it will be used to study the final version of the pulser (following).

The Pulser

Appendix A, and Table AI indicate that the requirements for the pulser and switch are:

1) Provide a pulse of width $(0 \rightarrow 0) \sim 85$ n.s. and a peak voltage of $\sim 40$ $\mathrm{kV}$.

2) Must switch $\sim 2.5 \mathrm{k} . \mathrm{A}$. in $\leqslant 20 \mathrm{n.s}$.

3) For high efficiency the flat top should be $\geqslant 20 \mathrm{n.s}$. In addition J. Peterson's calculation $(\mathrm{CN}-49)$ of the properties of penetration of magnetic fields through the thin metalic coating of the ceramic beam tube makes the flat top highly desirable.

The voltage needed, and the stability requirements on the power supply makes a sinple cable pulser (Mercury switch type) rather wasteful, since 
$\mathrm{V}$ (pulse) $=1 / 2 \mathrm{~V}$ (charging). It was decided to use a voltage doubler (Blumlein type). A prototype doubler made out of cables worked very well, but the pick up from the "spectator cable" was high. For this and other reasons 1 isted below and after many discussions with A. Odian and F. Villa, I decided to use a wrap around Blumlein doubler (see Figure 1).

Although a spark gap can switch a large amount of current fairly fast, the rep rate of $180 \mathrm{c} / \mathrm{s}$ makes such a switch undersirable. A high quality thyratron is needed. C. 01 son and $B$. Tomlin are responsible for the thyratron and power supply. Specs are already out and there are candidates. The power supply and thyratron will not be discussed here except to say that all thyratrons that can do the job are preferably oil immevsed for cooling and insulation. This makes oil also desirable as the Blumlein dielectric.

A Blumlein with polyethelene dielectric was constructed under the supervision of Jim Weaver. It will be used shortly to pulse the existing (prototype) magnet for comparison with the cable Blumlein.

The use of oil as dielectric is desirable for the following reasons:

1) The thyratron likes to be in oil any way.

2) The uncertainty in the natural $\mu, \varepsilon$ of the ferrite, and other end effects, translate into some uncertainty $(\sim 10 \%)$ in the impedance of the magnet. For matching the line to the magnet it is very easy to change the line impedance by simply changing the spacing at very little cost. We have to worry only about the cables.

The Final Magnet

To save on cost we are trying to use blocks of $4 \mathrm{C} 4$ Ferrite which $G$. Fischer has. These have dictated the spacing of the magnet electrodes. Their dimensions make the 2nd geometry in Table I a better choice. Combining all factors the parameters of the magnet are: 
1) $\mathrm{z}=16.7 \Omega$, over al1 length $<40 \mathrm{cms}$.

2) Ferrite length along the beam $=33 \mathrm{cms}$ the average length of the ferrite in cross section $=74 \mathrm{cms}$, the thickness $2 \mathrm{cms}$.

3) Magnet Gap width $=$ Gap height $=2.25 \mathrm{cms}$. (The beam pipe is $2.1 \mathrm{~cm} \mathrm{0.D.).}$

Figure 2. shows a sketch of the proposed magnet. It is very similar to the prototype. Figure 3 is the proposed arrangement of the pulser and magnet in the ring. It has some flexibility, and the best arrangement is under investigation.

\section{Other Details}

a) TERMINATION: $16.7 \Omega, \approx 1.5 \mathrm{k.W}$. R. Rwoe is looking at the type of cooling (air, oil, heat exchanger etc.), and what type of resistance would be best.

b) DRIVE CABLES: We have a usable $50 \Omega$ cable in stores. It is, however, large in diameter $\left(1^{\prime \prime}+\right)$ and somewhat stiff. I would like to suggest that, since there are several kickers and may be other uses for a good H.V. cable, and since cable manufacturers are getting smarter, users interested can share the cost of procuring a decent cable.

In any case, J. Broeder is working out a suitable "connector" for the cable we have, which can be attached to the magnet at any place desired. Considering the tightness of space this saves a lot of agony. 
Page 5
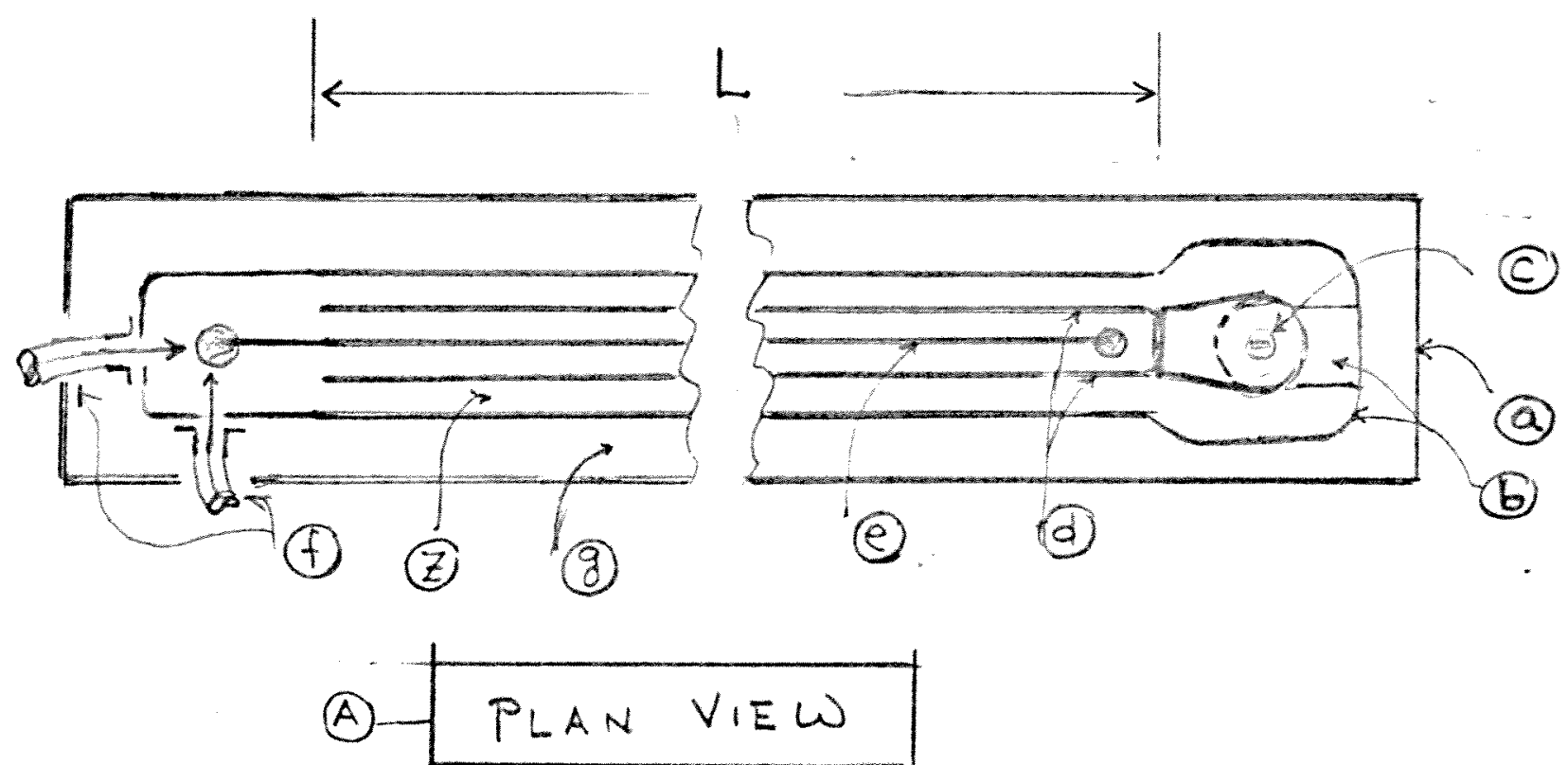

(a) OL TANK AND OL (g)

(b) GROUND ELECTRODE OF LINE AND GROUND CONNECTION TO THYRATRON. GRAD ELECTRODE IS COVERED TOR 2 BOTTOM

(c) THYRATRON \& CONNECTION TO H.V ELECTRODE (d)

(e) output electrode; (f) Possible cable connection TO magnet (cables can enter either side, END ON, OR BOTTOM.).

(c) active length. It depends on dielectric $\&$ PULSE WIDTH REQUIRED.

(2) SINGLE GAP IMPEDENCE = MAGNET IMPEDENCE

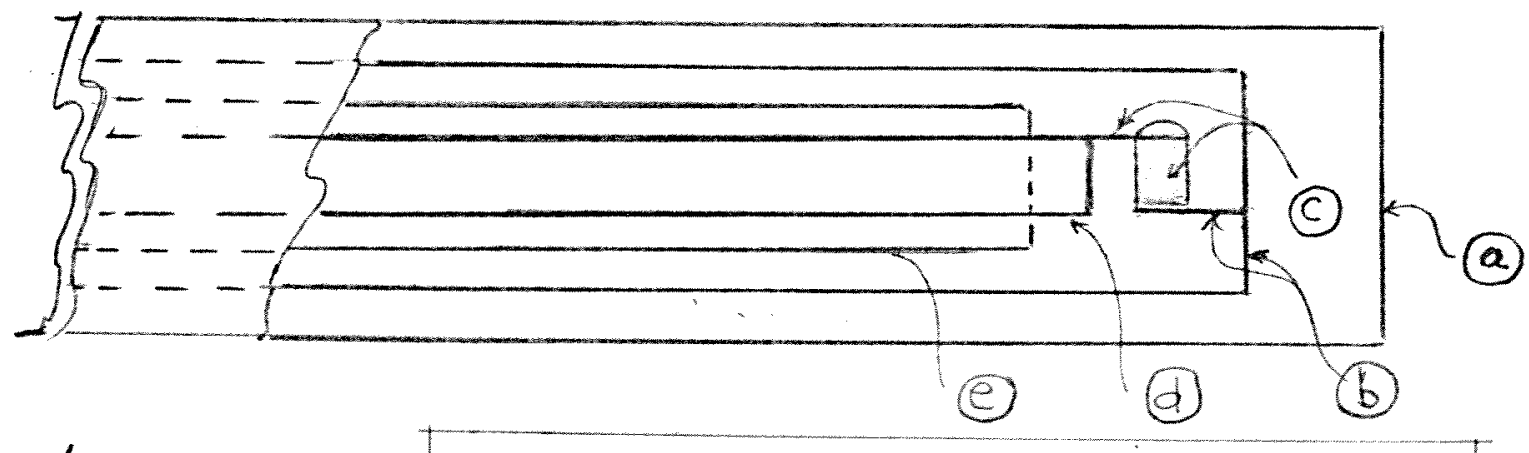

FIG 1.

(B)- SChEMATIC SIDE VIEL at Thyratron 
Page 6
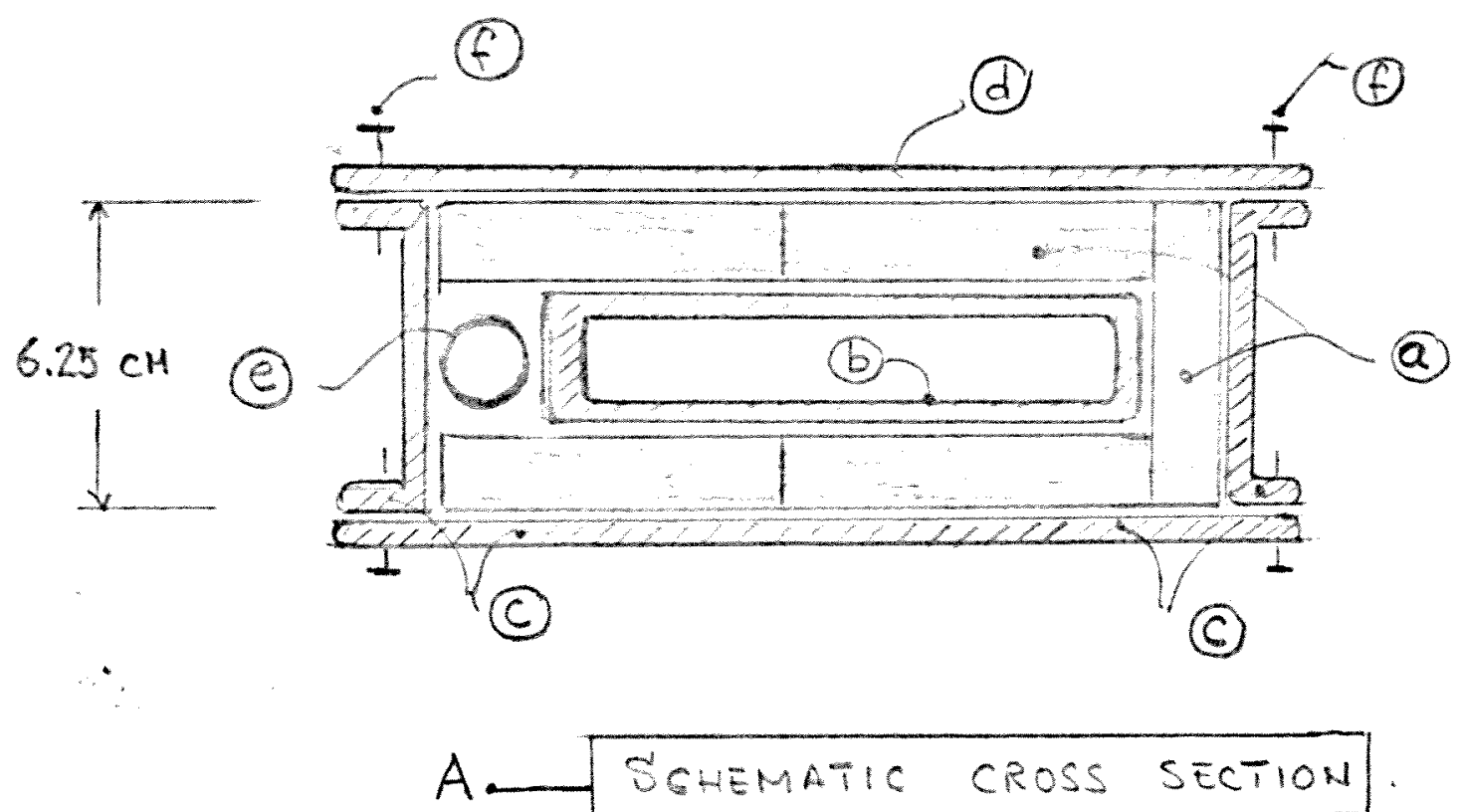

(a) FERRITE BLOCKS; (b) H.V ELECTRODE, OPEN BOX STRUCTURE; (C) BOTTOM AND SIDE GRAD ELECTRODE \& (d) TOP GRND ELECTRODE

(e) Beam tube; 9 Bolting arrangement

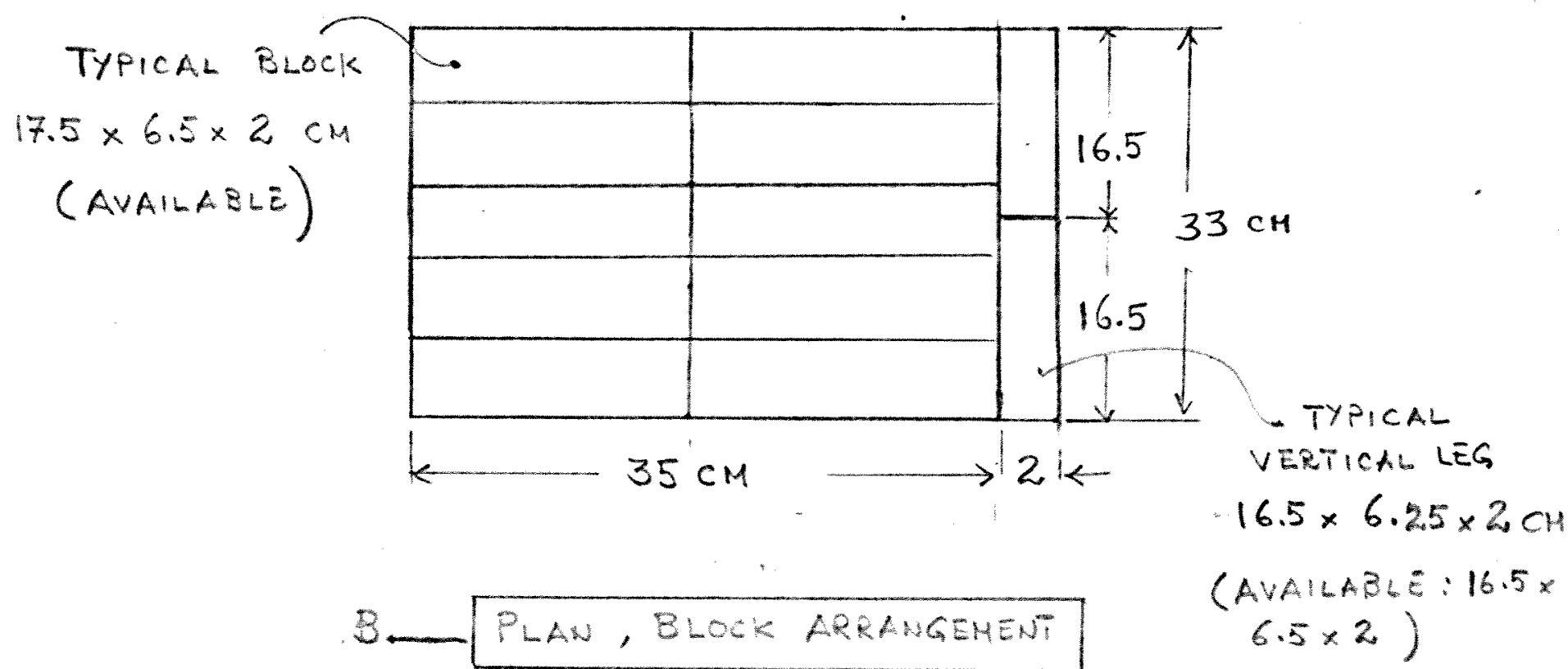

Fig 2. magnet geometry, schematic 
Fig 3

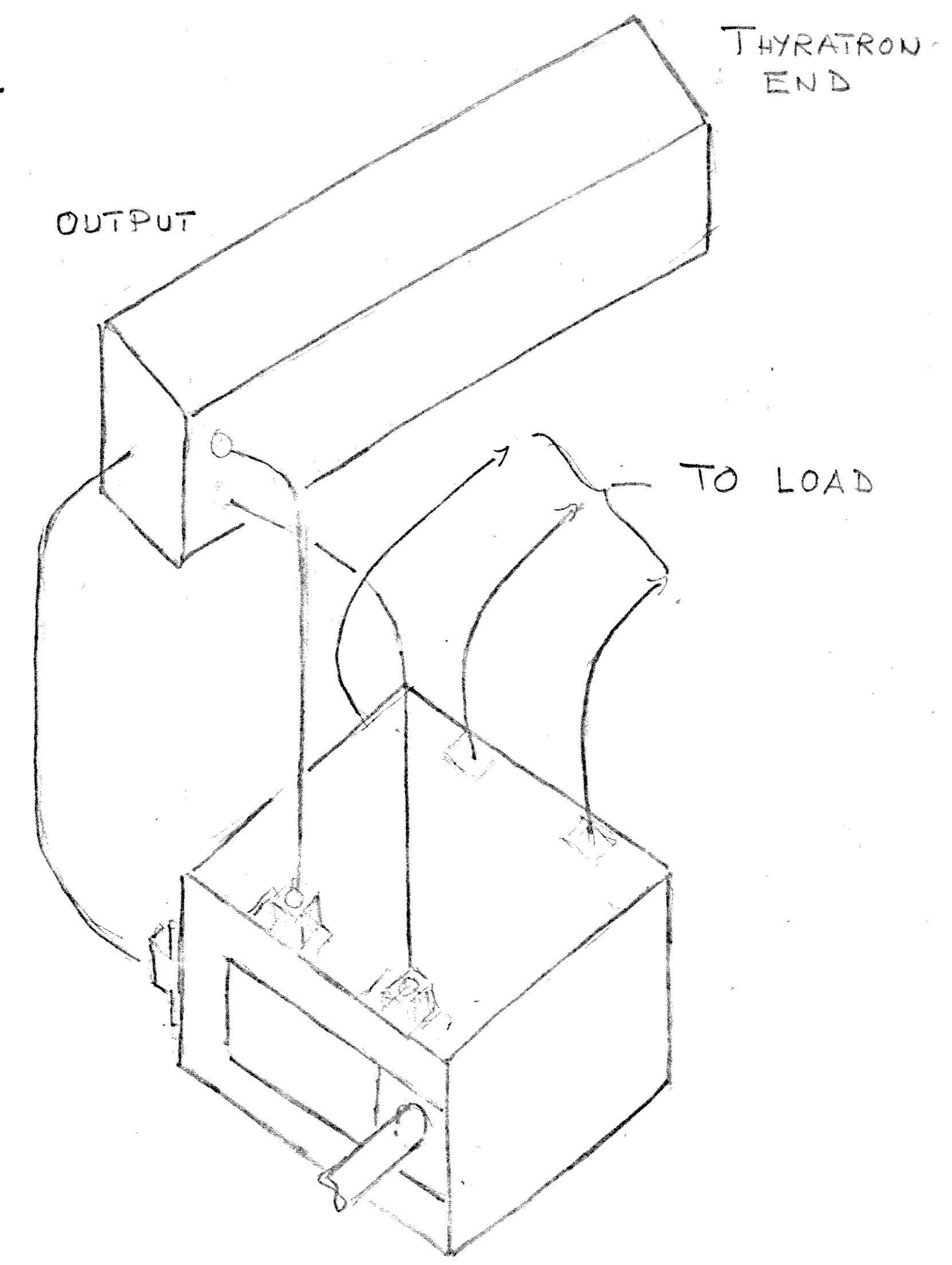




\section{APPENDIX A}

This analysis was written in December 1980 and copies were sent to B. Richter, G. Fischer, J. Peterson, B. Tomlin, C. O1son. At that time the thickness of the ferrite, the width of gap and its height were all $2 \mathrm{cms}$. Since then the beam tube O.D. changed slightly and the gap width and height were changed to $2.25 \mathrm{cms}$. This changes the value of the effective $\mu\left(\mu_{e}\right)$, the effective $\varepsilon\left(\varepsilon_{e}\right)$ and the required $B$ field.

However, the change is small and amounts to $\approx 10 \%$ increase in voltage. The power supply has a margin of safety $\approx 30 \%$.

Please note that the transit time, flat top, and pulse width at the base used in integrating the field (Fig. A.1) are just for convenience, and serve only to illustrate the principle

\section{Cooling Ring Ferrite Kickers}

A set of equations were used to design a ferrite test kicker. The performance was found to agree very well with calculations.

Define:

$$
\begin{aligned}
& \mathrm{S}(\mathrm{cm})=\text { Magnet length } \\
& \mathrm{Z}(\Omega)=\text { Impedance } \\
& \text { L (h) = Thyratron circuit inductance } \\
& t_{r}, t_{b}(n \cdot s)=\text { Rise time of electric } \\
& \text { pulse, width at base } \\
& \tau \text { (n.s.) }=\text { Pulse transit time in magnet } \\
& \ell, R(\mathrm{~cm})=\text { Ferrite cross-section length, radius if cylidrical } \\
& \omega(\mathrm{cm})=\text { Ferrite thickness } \\
& \mathrm{g}(\mathrm{cm})=\text { Gap }
\end{aligned}
$$


$\mu, \mu_{\mathrm{e}}=$ Permiability, effective perm.

B $($ Tesla $)=$ Magnetic field

$I($ K.A. $)=$ Current required

Then the equations used are:

$$
\begin{aligned}
& \mathrm{B}(\mathrm{g}+\ell / \mu) \quad=\mathrm{I} \mu_{\mathrm{o}} \ldots \ldots-\ldots-\ldots \\
& \left.\begin{array}{l}
\mu_{\mathbf{e}}=\mu(\ell+g) /(\mu g+l) \\
\varepsilon_{\mathbf{e}}=(\varepsilon l+g) /(\ell+g)
\end{array}\right\}-\cdots-\ldots-\ldots-\ldots \text { II } \\
& \sqrt{\mu_{\mathrm{e}} \varepsilon_{\mathrm{e}}} \times \frac{\mathrm{S}}{33}=\tau-\cdots-\cdots-\cdots-\cdots \text { - }-\cdots \text { III } \\
& \left.\begin{array}{l}
\sqrt{\mu_{\mathrm{e}} / \varepsilon_{\mathrm{e}}} \times 60 \times \ell_{\mathrm{n}} \frac{\mathrm{R}_{2}}{\mathrm{R}_{1}}=\mathrm{z} \text { (circular) } \\
\approx \sqrt{\mu_{\mathrm{e}} / \varepsilon_{\mathrm{e}}} \times 377 \times \frac{\omega}{\ell+g} \text { (closed square) }
\end{array}\right\} \\
& \frac{\mathrm{L}}{\mathrm{Z}}=\mathrm{t}_{\mathrm{r}}-\cdots-\cdots-\cdots-\cdots-\cdots
\end{aligned}
$$

Since transit time of particle in magnet is negligible, it sees a snap shot of the integrated field. The bunch we wish to kick should be at the magnet at the time when field is max. To avoid disturbing the second bunch, the field should go from max to zero between bunches.

As will be shown, the time ${ }_{0 \rightarrow 0}$ is $=t_{b}+\tau$, and the field reaches the max. possible if $\tau \leq$ pulse flat top.

Figure 1 illustrates the last two relations. Trapizoidal pulses were used for simplicity and the rather coarse field integration was done graphically. The values of $\tau, t_{r}$ are arbitrary and short for convenience. In 1.a $\tau=$ pülse flat top, in $1 . b \tau>$ pulse flat top. Le us summarize these relations:

$$
\left.\begin{array}{rl}
t\left(\begin{array}{l}
B \\
0 \rightarrow 0
\end{array}\right) & =t_{b}+\tau \\
t\left(\begin{array}{l}
B \\
\max \rightarrow 0
\end{array}\right) & =1 / 2\left(t_{b}+\tau\right) \\
& <\text { Interbunch time }
\end{array}\right\}-\cdots-\cdots-\cdots--V I
$$


$B$ reaches max. possible if

$\left.\begin{array}{rl}\tau & \leqslant \text { Pulse flat top } \\ & \leqslant t_{b}-2 t_{r}\end{array}\right\}-\cdots-\cdots-\cdots-\cdots-$ VII

\section{$\underline{\text { Practical Cases }}$}

You can convince yourself that in equations II, III and IV the magnet impedance $Z$ is determined by $l, g$, $\omega$. $S$ does not effect $Z$ as should be for a transmission 1ine. S effects $\tau$. If you fix $Z\left(t_{r}\right)$ and $\tau$, then you have to take whatever $S$ comes out. If you fix $z\left(t_{r}\right)$ and $S$, you have to accept whatever $\tau$ comes out.

The best approach is to fix the parameters which are dictated by other considerations.

In our case we want to utilize existing ferrite blocks, and we need a two cm gap.

$$
\text { : } \omega=\mathrm{g}=2 \mathrm{~cm} .
$$

The interbunch time $=50 \mathrm{n} . \mathrm{s}$. and space considerations make $\mathrm{s}=33 \mathrm{cms}$ practical.

$$
: \mathrm{s}=33 \cdot \mathrm{cm}, 1 / 2\left(t_{\mathrm{b}}+\tau\right)<50 \mathrm{n} \cdot \mathrm{s}
$$

\section{CASE A :}

$\mathrm{z}=16.7, \mathrm{t}_{\mathrm{r}}=20 \mathrm{n.s}$. A good thyratron can do this. The solution resulting is shown in Table I. $\tau$ turns out to be $16.5 \mathrm{n} . \mathrm{s}$. If $t_{b}=60$, the flat top $\approx 20$ then both equations VI, VII are fulfilled with some margin of safety. 


\section{CASE B $\quad$ :}

We try to reduce voltage by lowering impedance. Choose $Z=12.5$. Table I shows that the voltage is somewhat lower al though the current goes up. $\tau$ is $19 \mathrm{n.s}$. and even if $t_{\mathrm{r}}$ deteriorates to say $25 \mathrm{n} . \mathrm{s}$. We can still fulfill VI and VII safely. The ferrite cross-sectional length $\ell$ has increased from $74 \rightarrow 115 \mathrm{~cm}$, somewhat large. Inspite of the extra $5 \mathrm{kV}$ in the pulse I prefer Case A which is more compact. 